\title{
Childhood sexual trauma in women with interstitial cystitis/bladder pain syndrome: a case control study
}

\author{
J. Curtis Nickel, MD, FRCSC;, Dean A. Tripp, PhD, FRCSC, ${ }^{+}$Michel Pontari, MD," Robert Moldwin, MD, Robert \\ Mayer, MD; $;$ Lesley K. Carr, MD, FRCSC,; Ragi Doggweiler, MD; Claire C. Yang, MD: Nagendra Mishra, MD; ;t \\ and Jorgen Nordling ${ }^{\S \xi}$
}

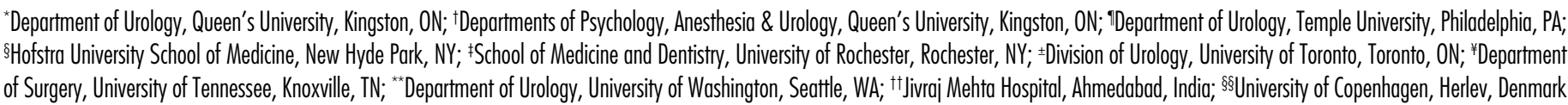

Cite as: Can Urol Assoc J 2011;5(6):410-5; htrp://dx.doi.org/10.5489/cuaj.11110

\section{Abstract}

Background: The impact of early lifetime trauma on symptom severity and quality of life of patients with interstitial cystitis/bladder pain syndrome (IC/BPS) has not been fully elucidated. We wanted to determine and compare the prevalence and impact of childhood traumatic events, with an emphasis on childhood sexual abuse, on patient symptoms, quality of life and other biopsychosocial parameters.

Methods: Subjects (female patients with IC/BPS and controls without IC/BPS) completed psychosocial phenotyping questionnaires, including a demographics/history form, and validated questionnaires focused on presenting symptoms (IC symptom indices, pain), psychosocial parameters (depression, anxiety, pain catastrophizing, sexual functioning, social support) and quality of life. Participants also completed the Childhood Traumatic Events Scale.

Results: Questionnaires were completed by 207 IC/BPS patients and 117 controls matched for age, partner status and education. It was found that before 17 years of age, the IC/BPS cases reported higher prevalence of "raped or molested" compared to controls $(24.0 \%$ vs. $14.7 \% ; p=0.047)$. Within the IC/BPS group, cases reporting previous sexual abuse endorsed greater sensory pain, depression and poorer physical quality of life at the present time compared to IC cases without a sexual abuse history. In the controls only, those reporting previous sexual abuse endorsed more depression, anxiety, stress, social maladjustment poorer mental quality of life in the present time. When the analysis was corrected for potential multiple comparison error, none of the findings remained significant in either the IC/BPS or control groups.

Interpretation: Childhood traumatic events, in particular sexual abuse and extreme illness, are reported as more common in IC/ BPS patients than controls. Early trauma, such as the occurrence of sexual abuse, is associated with some differences in patient adjustment (e.g., pain, quality of life, depression) but this impact appears to be, at most, very modest.

\section{Introduction}

Reports of childhood traumatic events, including sexual abuse, in patients with interstitial cystitis/bladder pain syndrome (IC/BPS) have engendered controversy and debate in the urology and IC/BPS patient community. ${ }^{1}$ It has been documented that patients diagnosed with IC/BPS have a higher prevalence of reported sexual abuse than women without IC/BPS ${ }^{2}$ and that the presenting symptoms of IC/ BPS patients reporting abuse are different than those without a history of abuse. ${ }^{3}$ This literature would suggest that this could be an important observation to expand our understanding of this enigmatic urologic chronic pelvic pain syndrome. Women who report a history of sexual trauma or abuse have increased rates of subsequent pelvic pain ${ }^{4}$ and vulvodynia. ${ }^{5}$ However, the chronic pain literature contains contradictory evidence on the importance of self-reported early or childhood abuse predicting greater pain severity or other pain-related impairment. ${ }^{6}$ To clarify the association of sexual abuse and IC/BPS, we used a large age-matched casecontrol multicentre questionnaire based survey to compare the prevalence and impact of childhood traumatic events, particularly sexual abuse, on patient symptoms, quality of life and other related biopsychosocial parameters.

\section{Methods}

\section{Subjects and study design}

We recruited English-speaking patients from 9 participating centres who fulfilled the diagnostic criteria described in the IC Data Base Study ${ }^{7}$ and the definition of IC/BPS described at the NIH Urologic Chronic Pelvic Pain Consensus Symposium (Baltimore, December 2007). Control females were included if they had no identified diagnosis of IC/BPS. Patient populations and recruitment strategies for this case-control 
questionnaire-based study have been previously published. ${ }^{8}$

Following initial contact (phone, personal contact or letter), interested IC/BPS patients and control subjects were mailed a package containing two copies of the informed consent (specific Institutional Review Board-approved consent for patients and control participants) and the packet of questionnaires. Patients and control subjects were asked to return a signed copy of the informed consent, as well as a completed set of questionnaires.

\section{Questionnaires}

Patients completed a demographic and history questionnaire. All participants were asked to complete the Childhood Traumatic Events Scale (CTES). ${ }^{9}$ The CTES allows respondents to report traumatic events that occurred before age 17. These traumatic events include sexual abuse (raped or molested), violence or abuse that is not sexual, parental divorce (or separation), extreme illness and/or death of a family member or friend. We assessed each question as follows: the age of trauma, perceived intensity of the trauma (on a scale of $1-7$, subjects rated "how traumatic" $1=$ not at all, 7 = extremely traumatic), and whether or not confiding in others occurred (on a scale where subject indicated they had confided in others; $1=$ not at all, $7=$ a great deal).

The IC/BPS symptoms were assessed using the IC symptom index (ICSI) and IC Problem Index (ICPI), ${ }^{10}$ also known as the O'Leary-Sant Questionnaire, which assesses symptom severity and problems associated with four IC associated issues (frequency, urgency, nocturia and bladder pain).

Pain was assessed with the short-form of the McGill Pain Questionnaire (MPQ-SF), ${ }^{11}$ which measures the quality of pain by asking patients to rate the intensity of 15 verbal descriptors of pain on a 0 to 3 rating scale. The Pain Rating Index (PRI) is comprised of two scores: a sensory pain score and an affective pain score.

Depressive symptoms were assessed using the Center for Epidemiological Studies Depression Scale (CES-D), ${ }^{12}$ a 20-item validated depression scale. Anxiety was assessed using the trait anxiety scale of the State-Trait Anxiety Inventory (STAI). ${ }^{13}$ This is a 20 item sub-scale from the longer 40 item instrument. Stress was assessed using the Perceived Stress Scale (PSS), ${ }_{1}^{14}$ a widely used instrument for measuring the degree to which situations in one's life are appraised as being stressful. Items assess how unpredictable, uncontrollable, and how overwhelming respondents find their lives. The Pain Catastrophizing Scale (PCS) was used to measure catastrophizing cognitions concerning pain. ${ }^{15}$

Female sexual functioning was assessed using the Female Sexual Functioning Inventory (FSFI). ${ }^{16}$ This brief index provides an overall metric of sexual functioning, and subscales for desire, arousal, orgasm, difficulty with lubrication, satisfaction, and pain.
The social support network of subjects was assessed using the Multidimensional Scale of Perceived Social Support. ${ }^{17}$ This scale assesses three aspects of perceived social support from friends, family and significant others.

Functional health status was assessed using the Medical Outcomes Study (MOS) Short-Form 12 Quality of Life Questionnaire (SF-12), ${ }^{18}$ which measures two domains of health status: physical (SF-12-PCS) and mental (SF12-MCS) component functioning.

\section{Data analyses}

We used Chi-Square models to examine the differences in the types of trauma reported by women suffering from IC/ BPS and asymptomatic controls before age 17 years. T-test models were used to analyze mean differences in pain, IC symptoms and psychosocial variables between sexual abuse reporters and non-reporters before age 17 years in women with IC/BPS and in sexual abuse reporters and non-reporters before age 17 years in asymptomatic control women. Analyses were corrected for inflated error by using the Bonferroni Correction, which acts to reduce the alphabased on the number of analyses conducted. This conservative correction is used to correct for biased tests in sets of analyses leading to too many results showing as significant. ${ }^{19}$

\section{Results}

Questionnaires were completed by 207 IC/BPS patients and compared to 117 controls. The groups were matched for age (case 49.6 vs. control 47.8 years; $p=0.279$ ), partner status $(72.7 \%$ cases vs. $65 \%$ control living with a partner; $p=0.146)$ and education (93.3\% of cases vs. $97.4 \%$ of controls reporting more than high school education; $p=0.108$ ).

The prevalence of traumatic events before 17 years of age was tallied (Table 1). The significant differences between cases and controls before 17 years were in the categories of raped or molested $(24.0 \%$ vs. $14.7 \% ; p=0.047)$ and extreme illness (25.1\% vs. $14.8 \% ; p=0.031)$. There was no significant difference when data was examined by country. The mean age of the reported sexual abuse event in the IC/ BPS group was 10.8 years ( \pm 4.4 years) and control group was 10.6 years ( \pm 4.1 years). The IC/BPS patients reported a trauma severity rating (on a scale of $1-7$ with 7 being extremely traumatic) of $6.1( \pm 1.4)$ compared to the control subjects who reported a score of $5.3( \pm 2.0)$ (difference not significant). Based on the CTES confiding score (on a scale of 0-7 with 7 being a "great deal"), most subjects indicated that they did not freely confide in others, with IC/ BPS patients confiding to others slightly less than control subjects $(1.6 \pm 1.2$ vs. $2.4 \pm 2.2 ; p=0.048)$.

Compared to IC patients who answered negatively, those who reported being raped or molested before 17 years 
Nickel et al.

\begin{tabular}{|c|c|c|c|}
\hline Trauma Domain & IC/BPS & Control & $p$ value \\
\hline Raped or molested & $\begin{array}{l}24.0 \% \\
(n=204)\end{array}$ & $\begin{array}{c}14.7 \% \\
(n=116)\end{array}$ & .047 \\
\hline Violence/abuse not sexual & $\begin{array}{c}12.7 \% \\
(n=204)\end{array}$ & $\begin{array}{c}7.8 \% \\
(n=116)\end{array}$ & .169 \\
\hline Parental divorce/separation & $\begin{array}{l}25.1 \% \\
(n=203)\end{array}$ & $\begin{array}{c}20.7 \% \\
(n=116)\end{array}$ & .369 \\
\hline Extreme IIIness & $\begin{array}{l}25.1 \% \\
(n=203)\end{array}$ & $\begin{array}{c}14.8 \% \\
(n=115)\end{array}$ & .031 \\
\hline $\begin{array}{l}\text { Death of family member or } \\
\text { friend }\end{array}$ & $\begin{array}{c}47.5 \% \\
(n=204)\end{array}$ & $\begin{array}{c}47.0 \% \\
(n=117)\end{array}$ & .926 \\
\hline
\end{tabular}

IC: interstitial cystitis; BPS: bladder pain syndrome.

reported greater pain (primarily sensory), depression and poorer physical quality of life (Table 2a). The IC/BPS patients who reported sexual abuse did not appear to suffer greater IC/BPS specific symptoms or sexual dysfunction than those who did not report the abuse. The control patients who reported being raped or molested compared to those who did not reported more depression, anxiety, stress, social maladjustment and poorer mental QoL (Table 2b). When appropriate statistical adjustments were made for the multiple comparisons, none of these associations remained statistically significant.

\section{Discussion}

Suggestions that childhood abuse could create a scenario in which pain could develop in the adult in the absence of a peripheral stimulus have been discussed for over 50 years. ${ }^{20} \mathrm{~A}$ recent meta-analysis was performed of retrospective reports of neglect, sexual or physical abuse experienced during childhood, and a measure of pain symptoms, chronic pain disorder status or health care visits. ${ }^{21}$ The authors found that adult patients with chronic pain are more likely to report abuse in childhood and that adults reporting a history of abuse in childhood also tend to report more current pain. On the other hand, these same authors found that the relationship between childhood abuse and pain is weak. The clinical significance of the relationship, particularly in urologic chronic pelvic pain such as that experienced in IC/ BPS, needs to be further explored. ${ }^{22}$

Sexual trauma in women aged younger than 17 is a social problem. It has been reported that almost $19 \%$ of children have been physically abused; $10 \%$ to $17 \%$ have been sexually abused. ${ }^{23}$ About 1 in 5 female high school students have reported being physically and/or sexually abused by a dating partner. ${ }^{24}$ Female veterans with lower urinary tract symptoms report higher prevalence of sexual trauma, including childhood abuse than a control population of veterans. ${ }^{25}$ Peters and colleagues documented that $17.7 \%$ of IC/BPS patients versus $8.2 \%$ of control subjects reported a history of sexual abuse. ${ }^{2}$ Clemens and colleagues ${ }^{26}$ found that women with IC in a population-based study were 9 times more likely to have a diagnostic code for child abuse (which includes sexual abuse, but also physical and emotional abuse) than agematched controls. In their selected cohort of women with IC/BPS, Seth and colleagues found that $25 \%$ of women who reported a history of sexual abuse appeared to have a different clinical presentation than those with no reported abuse history. ${ }^{3}$ Patients with IC/BPS and a history of sexual abuse had less frequency, nocturia, larger voided volumes, more tender areas on physical examination and worse domain scores on FSFI compared to those IC/BPS patients without a history of abuse. Both Peters and colleagues ${ }^{2}$ and Seth and colleauges ${ }^{3}$ have hypothesized that the physical trauma associated with early abuse may increase the likelihood of pelvic pain through neurogenic inflammation, as well as central sensitization.

We have previously shown that IC/BPS patients experience more pain, depression, stress, anxiety, catastrophizing and poorer sexual functioning and quality of life compared to the age-matched control group. ${ }^{8}$ While our large wellmatched case-control study did show a higher prevalence of sexual abuse prior to age 17 in IC/BPS patients $(24.0 \%)$ compared to asymptomatic control subjects $(14.7 \%)$, the difference in our collective sample only reached modest statistical significance $(p=0.047)$. Patients reporting childhood sexual abuse reported more pain, depression and poorer quality of life than patients not reporting such abuse. The maladaptive coping mechanism of catastrophizing, anxiety, stress, mental quality of life, sexual functioning or social support relationships in the IC/BPS group showed no differences. When the McGill pain descriptors were evaluated separately, the sensory component of pain was more predominant in the IC/BPS patients reporting sexual abuse compared to those without, perhaps adding some credibility to the other arguments regarding possible associated mechanisms., Similar to the weak association with depression and physical quality of life, these relationships with sexual abuse became nonsignificant when appropriate statistical adjustments were performed for multiple tests. We further noted that there appeared to be a similar weak impact of childhood sexual abuse on depression, anxiety, stress, mental quality of life, social interaction in the non IC/BPS control group; again, when the appropriate statistics were applied, these apparent associations were not statistically significant. Although our statistical correction shows no statistically significant differences in symptoms or psychosocial impact of abuse in subjects and controls with or without childhood abuse, this test does not prove that differences do not exist. The reality of the data is that significantly more patients with IC/ BPS reported abuse compared to controls and those who reported abuse demonstrated more pain, depression and poorer quality of life. 


\begin{tabular}{|c|c|c|c|c|c|}
\hline Domain & Measure & Reported sexual abuse & $\mathbf{N}$ & Mean (SD) & $p$ value* \\
\hline \multirow{6}{*}{ Pain } & \multirow{2}{*}{ SF-MPQ (Total) } & No & 141 & $17.70(11.09)$ & 0.038 \\
\hline & & Yes & 48 & $21.50(10.27)$ & \\
\hline & \multirow{2}{*}{ SF-MPQ (Sensory) } & No & 143 & $13.38(8.16)$ & 0.037 \\
\hline & & Yes & 48 & $16.23(8.05)$ & \\
\hline & \multirow{2}{*}{ SF-MPQ (Affective) } & No & 147 & $4.33(3.69)$ & 0.124 \\
\hline & & Yes & 48 & $5.27(3.50)$ & \\
\hline \multirow{2}{*}{ Catastrophizing } & \multirow{2}{*}{ PCS } & No & 150 & $20.57(13.08)$ & 0.148 \\
\hline & & Yes & 48 & $23.6010 .93)$ & \\
\hline \multirow{2}{*}{ Depression } & \multirow{2}{*}{ CES-D } & No & 140 & $17.52(13.70)$ & 0.046 \\
\hline & & Yes & 45 & $21.87(12.01)$ & \\
\hline \multirow{2}{*}{ Sexual functioning } & \multirow{2}{*}{ FSFI } & No & 114 & $17.39(10.73)$ & 0.317 \\
\hline & & Yes & 40 & $15.43(10.56)$ & \\
\hline \multirow{2}{*}{ Anxiety } & \multirow{2}{*}{ STAI } & No & 144 & $41.57(16.40)$ & 0.597 \\
\hline & & Yes & 47 & $42.98(13.67)$ & \\
\hline \multirow{4}{*}{ Quality of life } & \multirow{2}{*}{ SF-12 (Physical Come Scale) } & No & 143 & $41.84(8.52)$ & 0.009 \\
\hline & & Yes & 47 & $38.08(8.48)$ & \\
\hline & \multirow{2}{*}{ SF-12 (Mental Comp Scale) } & No & 143 & $44.49(9.26)$ & 0.366 \\
\hline & & Yes & 47 & $43.12(8.04)$ & \\
\hline \multirow{2}{*}{ Stress } & \multirow{2}{*}{ PSS } & No & 145 & $18.54(9.33)$ & 0.176 \\
\hline & & Yes & 49 & $20.51(6.75)$ & \\
\hline \multirow{2}{*}{ Social support } & \multirow{2}{*}{ MSPSS } & No & 143 & $65.74(17.85)$ & 0.052 \\
\hline & & Yes & 47 & $59.91(17.53)$ & \\
\hline \multirow{4}{*}{ IC symptoms and bother } & \multirow{2}{*}{ ICSI } & No & 150 & $12.06(4.99)$ & 0.431 \\
\hline & & Yes & 49 & $12.69(4.27)$ & \\
\hline & \multirow{2}{*}{ ICPI } & No & 152 & $10.48(4.35)$ & 0.345 \\
\hline & & Yes & 49 & $11.14(3.74)$ & \\
\hline
\end{tabular}

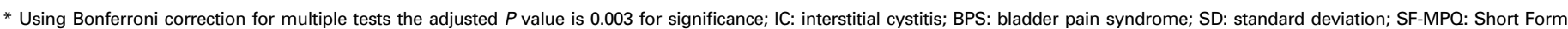

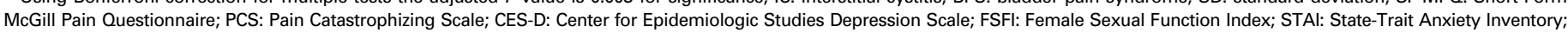
SF: short form; PSS: Perceived Stress Scale; MSPSS: Multidimensional Scale of Perceived Social Support; ICSI: interstitial cystitis symptom index; ICPI: interstitial cystitis problem index.

Do our findings conflict with the contemporary understanding of the relationship of childhood sexual abuse and chronic pain syndromes? We do not believe this is the case. The clinical utility of "biased" self-reporting of abuse, ${ }^{27}$ the very large sample size necessary in studies attempting to show such a relationship, ${ }^{22}$ the lack of prospective longitudinal studies ${ }^{28}$ and the fact that prospective, potentially less biased studies ${ }^{29,30}$ have failed to confirm such a relationship between childhood sexual abuse and chronic adult pain suggest that our study conclusions are indeed informative. However, a meta-analysis ${ }^{21}$ examining this question points to at least a weak association between childhood sexual abuse and chronic pain, one that becomes even weaker (but still statistically significant) when the analysis is controlled for depression. Since we noted a slightly higher prevalence of childhood sexual abuse in the IC/BPS group and weak associations with pain, depression and quality of life, we cannot rule out that for some women in this study, early-life trauma may have a strong and negative impact in regard to these or other un-examined variables.
While a major limitation in reported recollections of childhood events in our patients could have been a biased recall about abuse histories, this bias would likely be in favour of prevalence and psychological impact, rather than the other way around. Furthermore, questionnaire selfreports of sexual abuse may not be similar to that obtained by personal interview. When examined in isolation, reports of childhood sexual abuse in our study was not necessarily associated with significantly more present time pain, symptoms and maladaptation; however, it can be hypothesized that this early environment may predispose some patients to a more severe or a different form of IC/BPS in later years. Sexual abuse is rarely a one-time event, but rather a sustained exposure to abuse for most victims. In this sense, the early childhood environment is "hostile," and it is possible that these subjects grow up in an environment that is threatening in many respects (i.e., sexual abuse, domestic violence, lack of security) which results in higher incidence of early depression and poor coping mechanisms. This scenario makes it speculative to interpret the relationship to the 
Nickel et al.

\begin{tabular}{|c|c|c|c|c|c|}
\hline Domain & Measure & Reported sexual abuse & $\mathbf{N}$ & Mean (SD) & $p$ value* \\
\hline \multirow{6}{*}{ Pain } & \multirow{2}{*}{ SF-MPQ total } & No & 94 & $3.36(6.58)$ & \multirow[t]{2}{*}{0.778} \\
\hline & & Yes & 17 & $3.82(3.12)$ & \\
\hline & \multirow{2}{*}{$\begin{array}{l}\text { SF-MPQ (Sensory } \\
\text { Descriptors) }\end{array}$} & No & 95 & $2.63(4.93)$ & \multirow[t]{2}{*}{0.659} \\
\hline & & Yes & 17 & $3.17(2.60)$ & \\
\hline & \multirow{2}{*}{$\begin{array}{l}\text { SF-MPQ (Affective } \\
\text { Descriptors) }\end{array}$} & No & 95 & $.72(.02)$ & \multirow[t]{2}{*}{0.875} \\
\hline & & Yes & 17 & $.64(.86)$ & \\
\hline \multirow{2}{*}{ Catastrophizing } & \multirow{2}{*}{ PCS } & No & 96 & $9.39(8.77)$ & \multirow[t]{2}{*}{0.160} \\
\hline & & Yes & 17 & $12.82(11.42)$ & \\
\hline \multirow{2}{*}{ Depression } & \multirow{2}{*}{ CESD } & No & 95 & $6.45(7.44)$ & \multirow[t]{2}{*}{0.005} \\
\hline & & Yes & 17 & $12.58(11.52)$ & \\
\hline \multirow{2}{*}{ Sexual functioning } & \multirow{2}{*}{ FSFI } & No & 72 & $25.22(9.66)$ & \multirow[t]{2}{*}{0.984} \\
\hline & & Yes & 9 & $25.28(9.54)$ & \\
\hline \multirow{2}{*}{ Anxiety } & \multirow{2}{*}{ STAI } & No & 91 & $29.18(8.81)$ & \multirow[t]{2}{*}{0.043} \\
\hline & & Yes & 17 & $34.35(12.88)$ & \\
\hline \multirow{4}{*}{ Quality of life } & \multirow{2}{*}{ SF12 (Physical Comp Scale) } & No & 95 & $50.02(6.02)$ & \multirow[t]{2}{*}{0.089} \\
\hline & & Yes & 17 & $47.24(6.57)$ & \\
\hline & \multirow{2}{*}{ SF12 (Mental Comp Scale) } & No & 95 & $49.52(6.94)$ & \multirow[t]{2}{*}{0.036} \\
\hline & & Yes & 17 & $45.65(6.81)$ & \\
\hline \multirow{2}{*}{ Stress } & \multirow{2}{*}{ PSS } & No & 98 & 11.96 (5.99) & \multirow[t]{2}{*}{0.043} \\
\hline & & Yes & 17 & $15.29(7.16)$ & \\
\hline \multirow{2}{*}{ Social support } & \multirow{2}{*}{ MSPSS } & No & 95 & $71.66(13.27)$ & \multirow[t]{2}{*}{0.007} \\
\hline & & Yes & 17 & $61.82(18.02)$ & \\
\hline
\end{tabular}

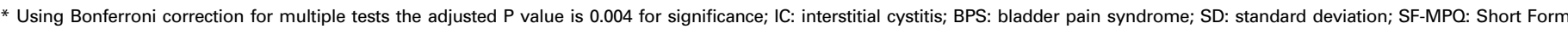

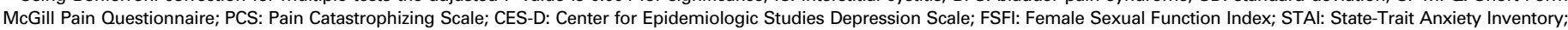
SF: short form; PSS: Perceived Stress Scale; MSPSS: Multidimensional Scale of Perceived Social Support; ICSI: interstitial cystitis symptom index; ICPI: interstitial cystitis problem index.

present-day psychosocial variables tested in this study. The strengths of our study included the appropriate selection of well-matched groups of IC/BPS patients and asymptomatic control subjects, the focus on childhood abuse (differentiated from recent abuse history), robust numbers in all comparison groups, enrollment from multiple sites, the comprehensive battery of psychosocial questionnaires administered and our statistical corrections for multiple tests. Replication and extension of these results and similar examination of recent abuse history are required in future research.

\section{Conclusions}

In the present sample, sexual abuse is reported more commonly in IC/BPS patients than controls, but such childhood abuse histories, at most, only moderately affect adult symptoms or sexual functioning.

Competing interests: None declared.

This paper has been peer-reviewed.

\section{References}

1. Allen P. Abuse and Interstitial cystitis: Is there a connection worth examining? Urology Times 2009;37:52.

2. Peters KM, Kalinowski SE, Carrico DJ, et al. Fact or Fiction—Is Abuse Prevalent in Patients With Interstitial Cystitis? Results From a Community Survey and Clinic Population. J Urol 2007;178:891-5.

3. Seth A, Teichman JMH. Differences in the Clinical Presentation of Intersititial Cystitis/Painful Bladder Syndrome in Patients With or Without Sexual Abuse History. J Urol 2008;180:2029-33.

4. Chandler H, Ciccone DS, Raphael KG. Localization of Pain and Self-Reported Rape in a Female Community Sample. Pain Med 2006; 7:344-52.

5. Harlow BL, Stewart EG. Adult-onset vulvodynia in relation to childhood violence victimization. Am J Epidemiol 2005;161:871-80.

6. Raphael KG. Childhood Abuse and Pain in Adulthood More Than a Modest Relationship? Clin J Pain 2005;21:371-3.

7. Hanno PM, Landis JR, Matthews-Cook Y, et al. Diagnosis of Interstitial Cystitis Revisited: Lessons Learned from the National Institutes of Health, Interstitial Cystitis Data Base Study. J Urol 1999;161:553-7.

8. Nickel IC, Tripp DA, Pontari M, et al. Psychosocial Phenotyping of Women with IC/BPS: A Case Control Study. J Urol 2010;183:167-72.

9. Pennebaker JW, Susman JR. Disclosure of Traumas and Psychosomatic Procesess. Soc Sci Med 1988:26:327-32.

10. O'Leary MP, Sant GR, Fowler FJJ, et al. The interstitial cystitis symptom index and problem index. Urology 1997;49:58-61.

11. Melzack R. The Short-Form McGill Pain Questionnaire. Pain 1987;30:191-7.

12. Radloff L. The CES-D scale: A self-report depression scale for research in the general population. Appl Psychol Meas 1977;1:385-401.

13. Speilberger CD, Gorusch RL, Lushene R, et al. Manual for the State-Trait Anxiety Inventory (Form Y1). Palo Alto, CA: Consulting Psychologists Press, 1983. 
14. Cohen S, Kamarck T, Mermelstein R. A global measure of perceived stress. J Health Soc Behav 1983;24:385-96.

15. Sullivan MJL, Bishop SR, Pivik J. The Pain Catastrophizing Scale: Development and validation. Psychol Assess 1995;7:524-32.

16. Rosen R, Brown C, Heiman J, et al. The Female Sexual Function Index (FSFI): a multidimensional selfreport instrument for the assessment of female sexual function. I Sex Marital Ther 2000;26:191-208.

17. Zimet GD, Dahlem NW, Zimet SG, et al. The multidimensional scale of perceived social support. J Pers Assess 1988;52:30-41.

18. Ware J, Kosinski M, Keller SD. A 12-1tem Short Form Health Survey: construction of scales and preliminary tests of reliability and validity. Med Care 1996;34:220-33.

19. Tabachnick BG, Fidell LS. Using Multivariate Statistics, 3rd ed. New York: Harper Collins; 1996:100-85.

20. Engel GL. "Psychogenic" pain and the pain-prone patient. Am J Med 1959;26:899-918.

21. Davis DA, Leuken U, Zautra AJ. Are Reports of Childhood Abuse Related to the Experience of Chronic Pain in Adulthood? A Meta-analytic Review of the Literature. Clin J Pain 2005;21:398-405

22. Raphael KG, Chandler HK, Ciccone DS, et al. Is childhood abuse a risk factor for chronic pain in adulthood? Curr Pain Headache Rep 2004;8:99-110.

23. Gorey K, Leslie D: The prevalence of child sexual abuse: integrative review adjustment for potential response and measurement bias. Child Abuse Negl 1997;21:391-8.

24. Silverman JG, Raj A, Mucci $L A$, et al. Dating violence against adolescent girls and associated substance use, unhealthy weight control, sexual risk behavior, pregnancy, and suicidality. JAMA 2001;286:572-9.
25. Klausner AP, Ibanez D, King AB, et al. The Influence of Psychiatric Comorbidities and Sexual Trauma on Lower Urinary Tract Symptoms in Female Veterans. J Urol 2009;182: 2785-90.

26. Clemens JQ, Meenan R, O'Keefe Rosetti MC, et al. A Case-Control Study of Medical Comorbidities in Women With Intersititial Cystitis. J Urol 2008; 179:2222-5.

27. Hardt J, Rutter M. Validity of adult retrospective reports of adverse childhood experiences: review of the evidence. J Child Psychol Psychiatry 2004:45:260-73.

28. Widom CS, Raphael KG, DuMont KA. The case for prospective longitudinal studies in child maltreatment research: commentary on Dube, Williamson, Thompson, Felitti, and Anda (2004). Child Abuse Negl 2004;28:715-22.

29. Linton SJ. A prospective study of the effects of sexual or physical abuse on back pain. Pain 2002;96:34751.

30. Raphael KG, Widom CS, Lange G. Childhood victimization and pain in adulthood: a prospective investigation. Pain 2001;92:283-93.

Correspondence: Dr. J. Curtis Nickel, Professor of Urology, Queen's University, Canada Research Chair in Urologic Pain and Inflammation, Department of Urology, 76 Stuart St., Kingston General Hospital, Kingston, 0N K7L 2V7; fax: 613-545-1970; jn@queensu.ca 